\title{
Modelling Ecological Cognitive Re- habilitation Therapies for Building Virtual Environments in Brain Injury
}

\author{
J. M. Martínez-Moreno ，P. Sánchez-González ，M. Luna ， I. Roig , J. M. Tormos \\ E. J. Gómez
}

Keywords

Neurorehabilitation, cognitive rehabilitation, ecological rehabilitation, virtual rehabilitation, interactive video, eye-tracking, activity of daily living, brain injury

\section{Summary}

Background: Brain Injury (BI) has become one of the most common causes of neurological disability in developed countries. Cognitive disorders result in a loss of independence and patients' quality of life. Cognitive rehabilitation aims to promote patients' skills to achieve their highest degree of personal autonomy. New technologies such as virtual reality or interactive video allow developing rehabilitation therapies based on reproducible Activities of Daily Living (ADLs), increasing the ecological validity of the therapy. However, the lack of frameworks to formalize and represent the definition of this kind of therapies can be a barrier for widespread use of interactive virtual environments in clinical routine.

Objectives: To provide neuropsychologists with a methodology and an instrument to design and evaluate cognitive rehabilitation therapeutic interventions strategies based on $A D L s$ performed in interactive virtual environments.
Methods: The proposed methodology is used to model therapeutic interventions during virtual ADLs considering cognitive deficit, expected abnormal interactions and therapeutic hypotheses. It allows identifying abnormal behavioural patterns and designing interventions strategies in order to achieve errorless-based rehabilitation.

Results: An ADL case study ('buying bread') is defined according to the guidelines established by the $A D L$ intervention model. This case study is developed, as a proof of principle, using interactive video technology and is used to assess the feasibility of the proposed methodology in the definition of therapeutic intervention procedures.

Conclusions: The proposed methodology provides neuropsychologists with an instrument to design and evaluate ADL-based therapeutic intervention strategies, attending to solve actual limitation of virtual scenarios, to be use for ecological rehabilitation of cognitive deficit in daily clinical practice. The developed case study proves the potential of the methodology to design therapeutic interventions strategies; however our current work is devoted to designing more experiments in order to present more evidence about its values.

\section{Introduction}

Brain injury (BI) is a life-altering episode that may affect every area of a person's life including his/her relationship with family members and close relatives [1]. BI refers to medical conditions that occur in the brain, altering its function. These conditions include stroke, traumatic brain injury, tumour and other brain-related diseases $[2,3]$.

Cognitive processes determine individual performance in Activities of Daily Living (ADL) [4]. Thus, cognitive disorders after $\mathrm{BI}$ result in a loss of autonomy and independence, affecting the patient's quality of life [5]. Cognitive rehabilitation seeks to increase patients' autonomy and quality of life minimizing or compensating functional disorders showed by BI patients [6].

Brain plasticity is an intrinsic property of the human nervous system whereby its structure is changed depending on experience [7]. New neural connectivity patterns are continuously being established by these structural changes during a person's life. BI can disrupt neural connectivity and induce deficits; however brain plasticity remains on preserved brain areas. Neurorehabilitation pursuits a precise modulation of this intrinsic property, based on specific exercises to induce functional changes, which could result in partial or total recovery of the affected functions [8-10].

After a neurological damage, brain computational capacity is restricted, but the resulting instability in brain structure induces abnormally high capacity for change. To take advantage of this, therapeutic interventions should be as effective as possible. Benefits of rehabilitation treat- 
ments focused on specific cognitive functions (e.g.: attention, memory or executive functions) have been proved, but this evidence is more limited in terms of improving the level of functional activities, participation, or life satisfaction after cognitive rehabilitation [11]. Ecologically valid treatments, (i.e.: those that have a degree of relevance or similarity to the "real" world [12]), may enable not only cognitive functions' improvement, but also generalization and transfer of skill acquisition into reallife situations.

Adapting training cognitive strategies for experimental and active learning in relevant settings encourages motivation and facilitates the transfer to the real world [ 13 , 14]. Scientific literature has been published reporting the incorporation of behavioural approaches into cognitive rehabilitation programs [15]. These studies discuss the several reasons why behavioural methods are suitable and effective for treatment of people with BI. These strategies are concerned both with establishing or improving appropriate behaviours, and decreasing or eliminating inappropriate ones (for example in real-life situations). The disadvantages of these methods are the associated cost of therapeutic interventions and manpower, and the difficulties to monitor the patient's performance. However, there exist technological solutions for those rehabilitation treatments focused on specific cognitive functions that allow improving the rehabilitation process efficiency [16], as well as generating new clinical knowledge $[17,18]$. This would suggest that appropriate technology could be applied to cognitive, economically-sustainable strategies based on behavioural methods allowing therapeutic tracking and evaluation.

Video clips, virtual reality, or even cartoons are processed by humans as a representation of real live situations. This is an intrinsic property of human cognitive processing [19]. Recent studies show that brain structure used in processing this kind of information is similar than those used by processing real live experiences [19]. Virtual environments can be used to reproduce daily situations, which allow simulating controlled behavioural requirements similar to real life, therefore enhancing ecological validity of cognitive performance
[12]. Continuous advancements in computer processing, graphics and image rendering, display systems or tracking devices make the technology feasible for re-creating rehabilitation therapies based on $\mathrm{ADL}$. In these virtual environments the therapist is allowed to interact with patients without even being present, inhibiting unsuitable behaviour patterns, stimulating correct answers throughout the simulation and enhancing stimuli with supplementary information when necessary.

There are different approaches to the use of therapeutic virtual environments reported in the literature: a) reproducing specific daily situations like street crossing [20] or preparation of a hot drink [21]; b) focusing on training applied cognitive skills like spatial abilities in a route learning task [22] or attention, distraction and anxiety in a school class [23]; and c) creating complex environments (library [24], museum [25], kitchen [26-28], city [29-31] or a mall [32-35]) combining different activities. Regardless of the strategy, these studies highlight clinical potential of using ADLs for rehabilitation, interacting and reproducing daily and familiar situations to the patient.

Despite their therapeutic potential and advantages (safety, monitoring capabilities, repetitions, personalized difficulty, etc.) and the technological maturity achieved, there is still reticence to incorporate interactive virtual environment based therapies in daily clinical practice. Moreover, the evidence that the use of virtual reality in rehabilitation of traumatic brain injury improves cognitive functionality is currently very limited, as well as the improvement provided over the use of traditional therapies [36]. Taking this in mind, this research work hypothesizes two possible causes keeping interactive virtual environments outside the therapeutic clinical routine: (a) the absence of a methodology to conceptualize and formalize programs of intervention based on ADLs; and (b) the lack of representation tools of implicit knowledge in these treatments. Thus, this research work aims to provide neuropsychologists with the methodology and the instruments required to design ADL-based interventions strategies to be performed in interactive virtual environments.
The study is structured in the seven different sections, including the introduction. Section 2 first provides a brief overview of the related work. In Section 3 the requirements of an effective therapy based on interactive virtual environments are examined to analyse how they determine the ADL-based therapeutic intervention process' design. In Section 4, the proposed methodology is presented by describing the ADL intervention model. The model is represented by proposing an ontology and implemented with a graphical tool in order to support neurophysiologists. The proposed methodology is applied in the definition of an ADL in the case of study described in Section 5. Finally, Sections 6 and 7 present a brief discussion and the study conclusions respectively.

\section{Related Work}

Reproducing real environments to support therapeutic interventions requires the use of sophisticated multimedia technologies such as virtual reality. This kind of technological solutions has reached a standard of excellence in gaming industry; however, clinical applications require more detailed and controlled user experience. In a clinical context, therapists should propose situations and patients should provide answers, by mean of interacting with the scenario. Nowadays, solutions such as NeuroVR [37] try to bring clinical experts closer to the development of the activities. This platform allows them to adapt predefined virtual environments using libraries of multimedia elements. On the other hand, tools such as AGATHE [31] have their own interfaces to adjust level or feedback configuration of their predefined activities.

However, while this approach has been proved useful to generate successful proof of principle about the clinical utility of this technology, their impact in clinical routine is very limited. Moreover non-technological skilful clinicians do not find these applications appealing. In their work Lange et al. [38] detected the need of providing clinicians with toolkits which allow them to create and control interactive game-based rehabilitation tasks. 
Interactive virtual environments are comprised of different stimuli sources that introduce a high degree of behavioural requirements for the patient. The number of variables that can interfere in the most simple activity of daily live can easily scale up to the order of hundreds, when we consider all multiple interactions among them. This is not relevant from the user experience point of view in entertainment applications, but it remains a keystone for the therapeutic use of this technology. In order to determine the full potential of $\mathrm{ADL}$ based rehabilitation, one must avoid a "black box" mentality when analysing these systems. Active ingredients of each interaction must be specified, including the identification of therapeutic components contained therein and each one's role in the patient's behaviour [39].

Some authors emphasise the importance of working with well-defined rehabilitation therapies. Whyte et al. suggested the rehabilitation is in need of developing protocol-based treatments and tools to objectively verify their contents [39]. They have been developing a rehabilitation treatment taxonomy (RTT) based on treatment theory with the conviction that it will lead to a better understanding of rehabilitation interventions and their effects $[40,41]$. Grove developed an ontology for traumatic brain injury rehabilitation with the aim of providing an intermediate support for further ontologies development. Grove attached importance to the use of this kind of ontologies to improve clinical decisionmaking in traumatic brain injury rehabilitation by means of facilitating domain knowledge interoperability [42]. Thus, knowledge management languages and tools such as thesaurus, taxonomies or ontologies may help to apply and represent knowledge implicit in therapeutic activities.

Along the same line, but from a global perspective, Giustini et al. [43] highlight the important critical issue of correlating the real needs of rehabilitation practice with appropriate responses on the part of technological research. They suggest the need of developing new tools with which to study and clarify therapeutic intervention procedures, learning recovery and evidence in clinical activities; guaranteeing homogeneity and measurability of treatments, as well as the respective effects and functional results. The key aspect, also suggested by other authors [44], is to assess the efficacy and efficiency of interventions and to disseminate these new clinical strategies.

From the critical analysis of all these reported works, it seems reasonable to think about a graphical tool to describe the interactions with each element of the virtual scenarios. Built over a taxonomy of elements representing the clinical interventions, this tool will become a useful instrument to design rehabilitation virtual scenarios. Clinical experts will allow to conceptualize therapies based on these scenarios, which could be prescribed later by other clinicians according to the impairment profile of each subject.

\section{Analysis of Requirements}

To define cognitive rehabilitation strategies, we recruited a cohort of neuropsychologists with an outstanding background in the use of information and communications technology in neuropsychological rehabilitation. We conducted a bottom up analysis from the point of view of clinicians in order to define the pipeline of therapeutic intervention design. With that purpose, three workshops were organized with the participation of six neuropsychologists from Institut Guttmann Hospital. These working sessions analysed the complete ADL-based therapeutic intervention process, from virtual rehabilitation environments where they are reproduced to activities design.

The first goal was to identify the features that an interactive virtual environment must meet in order to become an effective resource for cognitive rehabilitation therapy. These characteristics were extracted analysing good rehabilitation process requirements and matched with assets provided by virtual reality or interactive video technologies: a) simple, established and easy interactions within an environment that does not require extra cognitive effort; b) stimulating, well defined stimuli and triggering responses to support patient in the ADL; c) supervised, monitored tasks; d) safe, errorless learning; e) asynchronous, virtual therapeutic interventions with no on-site supervision; and f) sustainable exploitation strategies.

Designing asynchronous and distance interventions require therapists to: a) hypothesise about possible responses of the patients in every situation, b) draw a plan for modelling their behaviour, designing therapeutic interactions for each patient's mistake, and c) define how the activity's flow is going to continue, in order to guide patients through different scenes towards a successful goal. Thus, the activity will be defined based on therapeutic hypotheses, which will be assessed with every patient's performance.

A successive approximation model was chosen as guideline for the ADL's structure [15]. The ADL can be divided into as many scenes as necessary. Each of these scenes is identified with a short-term goal which must be achievable by the patient. To complete the ADL, the patient must achieve every single short-term goal. In order to reach each short-term goal, the patient needs to interact with the elements shown in the scene. This presents an opportunity for the therapist to perform a therapeutic intervention on his/her behaviour. Therefore, neuropsychologists not only have to define the $\mathrm{ADL}$ but also have to plan their interventions to every possible patient's interaction.

\section{ADL Intervention Model}

\subsection{Model Conceptualization}

The proposed methodology addresses the ADL-based therapeutic intervention process design centred on the active elements of the treatment, planning the intervention strategies according to hypotheses about the patient's behaviour. Consistent with that purpose, an ADL definition model was developed. Firstly, three basic elements that allow defining therapeutic interventions were identified (i.e. Stimuli, Actions and Response Stimuli), and then model structure was defined according to the aforementioned requirements.

The Stimulus is the minimum unit of information used to elicit patient's reactions. An ADL is made of organized sets of Stimuli. Not only the elements that com- 
prise the activity's stages are stimuli, but also texts, audios, images, video clips, etc. used to enhance user experience. A Stimulus is defined by two parts, a descriptive one which identifies it as an object (i.e. the Stimulus is a door), and the second part which adds information about the state of the object (i.e. the door is open). Each Stimulus' state can be associated with a set of Actions.

The Action is another basic element which, linked to an interaction, can be defined as the patient's response to the Stimuli shown. Hence, Action provides patient interaction with a meaning within the $\mathrm{ADL}$ (i.e. a mouse click interaction associated with the "close" Action will change the state of the stimulus "door" from "open" to "close").

Action consequences in the activity are shown by other Stimuli called Response Stimulus. These Stimuli are triggered by a patient's interaction and allow therapists to give patients some feedback in order to support, correct, help or intervene in their behaviour (i.e. more direct instructions, highlighted Stimuli, encouraged messages, etc.).

Therefore, ADLs are made up by sets of Stimuli; changes in these sets are performed according to Action and Response logic; and shifting between Stimuli sets is triggered as a consequence of patient interaction. This allows representation of ADLs as a model based on events and states. States are Stimuli sets used to reproduce daily and familiar situations; and events are inputs to the states which correspond to patient's interactions. Such events induce changes from one state to another defining the rules that model the ADL.

With the aim of formalising the activities' definitions, the $\mathrm{ADL}$ model has been organised in a four level hierarchical structure ( $\triangleright$ Figure 1$)$ :

- ADL: Activity of Daily Living.

- Task: sub activities in which an ADL is divided.

- TaskStates: situations that have to be resolved by a patient to complete a Task.

- Pathway: sets of Stimuli, Action and Response Stimuli which define TaskStates.

The ADL has a main goal, for instance "buying bread", "getting dressed", "making something to eat", etc. Completing the activity requires performing a series of sub activities named Tasks.

A Task is considered an activity simpler than an ADL that preserves its own semantic identity. Performance in a Task is driven by an essential goal ("leave home", "arrive to the bakery", etc.) required to complete the ADL successfully. According to the successive approximation model every Task is divided into TaskStates, segments of the Task which are associated to short-term goals ("switch off the TV", "leave the sitting room", "open the drawer", etc.), and require patient interaction in order to achieve it.

TaskStates are defined by Pathways which allow therapists to: a) control the Stimuli showed to the patient every instant, b) determinate how he/she interacts with them, and c) define the feedback associated to each interaction.

Pathways are used to define the ADL according to the patient's behaviour. The neuropsychologist hypothesises about the patient's reactions to every set of Stimuli and the necessary therapeutic intervention to allow him/her to complete the ADL. Based on them, the neuropsychologist plans the required assistance that the patient will need according to his/her cognitive deficits.

In order to make ADL representation easier to handle, Pathways are classified attending to the contained information. Thus, four Pathways Representation Levels are defined depending on their implicit knowledge:
- First Representation Level includes all possible interactions that could be performed according to the Stimuli comprising a TaskState. Thus, Pathways are only related with the actions available in each Stimulus.

- Second Representation Level distinguishes between admissible and nonadmissible Pathways. Admissible Pathways are those which are defined as correct in order to reach the established goal. Non-admissible Pathways, on the other hand, refer to those interactions that prevent the patient from reaching the goal.

- Third Representation Level corresponds to therapeutic proposals for clinically intervening in the patient's behaviour. This is the selection of interactions that could help the patient to reach the goal depending on his/her expected wrong interactions. Thus, using this interventions neuropsychologists hypothesise about the patient's behaviour in every single ADL situation. Each time the patient performs an activity, the hypotheses will be evaluated to see whether they are successfully fulfilled.

- Fourth Representation Level corresponds to the patient's performance. This level collects the track of the patient through the $\mathrm{ADL}$, i.e. his/her interactions and the interventions he/she needed during ADL execution. Therefore, such Pathways represent the actual ADL performance of the patient contrasted with the neuropsychologist's hypotheses.

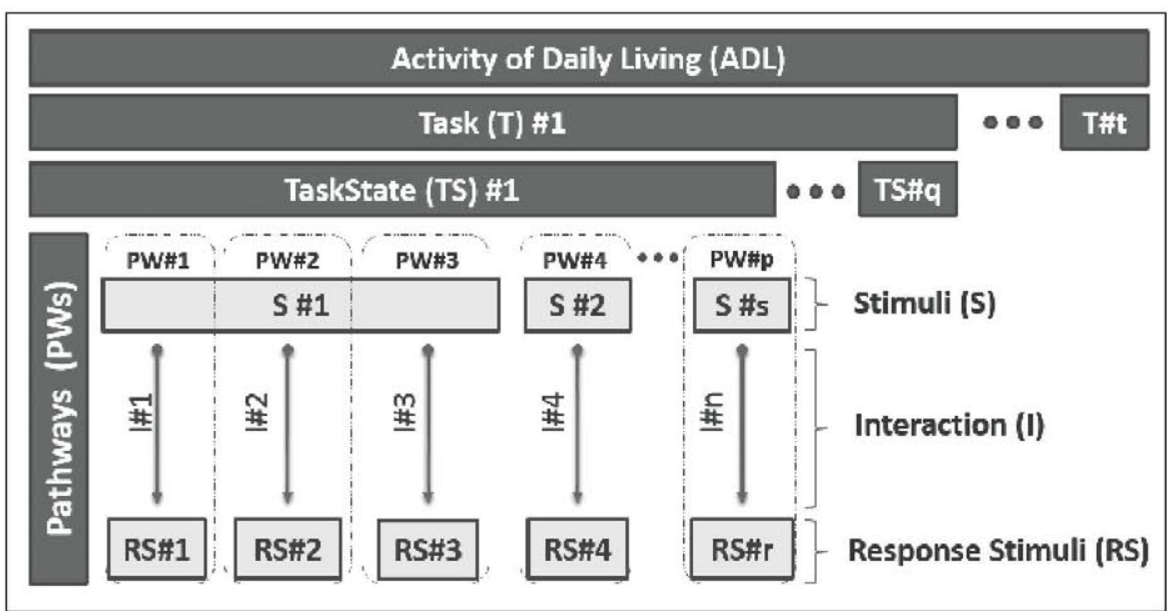

Figure 1 ADL hierarchical structure 


\subsection{Model Representation}

With the aim of providing a formal and explicit specification of the knowledge model an ontology for ADL-based therapeutic interventions domain was built using Protégé tool [45] and OWL 2 [46] as ontology language. This language is widely used as standard for representing and sharing knowledge and it is part of the $\mathrm{W} 3 \mathrm{C}$ recommendations related to the Semantic Web [47].

The model was adapted in order to be consistent with the ontology language rules. For instance, new elements were define such as "Leap" that represents the events involving changes in the activity flow, or "Interaction", which represents the means that patients have to interact with the activity.

As shown in Figure 2, the ontology classes and relationships were organized around two representation levels: (a) structural level representing the ADL map, providing a formalised description of the activity's elements and relationships ( $\$$ Figure 2a); and (b) the conceptual level representing the ADL from the therapeutic interventions' perspective ( Figure $2 \mathrm{~b}$ ).

\subsection{Model Implementation}

A graphical tool was developed in order to support neuropsychologists in the definition, creation and edition of ADLs based on the proposed methodology [48]. The design of the graphical tool was carried out following the principles of User Centred Design (UCD). The functional requirements were analysed by an expert panel of neuropsychologists and engineers from the clinical workshops involved in this research.

The final implementation was based on a Model-View-Controller (MVC) framework (Symfony2) and ORM Doctrine in the server side ( Figure 3). Extensive use of new functionalities provided by HTML 5 combined with JavaScript was used for interaction management in the client side. For a complete description the reader is referred to [48].

\section{Case Study}

With the aim of assessing the designed methodology, neuropsychologists from Institut Guttmann Hospital designed the
ADL 'Buying Bread' according to the guidelines drawn by the ADL model. The ADL was afterwards implemented using interactive video technology [49].

The ADL reproduces the steps that a subject, who is initially sitting on the sofa, has to follow in order to buy bread. Three Tasks corresponding to the three different stages where the activity is performed were identified: "home", "street" and "bakery". Task's goals, which were associated to each Task were: "picking up personal belongings and leaving home", "going to the bakery", and "buying bread", respectively.

Tasks were split into short-terms goals, TaskStates, which reproduced every single action that a person could do in a real life situation to reach the Task's goal. For example, "turning off the TV", "leaving the sitting room", "picking up keys and wallet", and "leaving home" are the short-term goals identified in the Task "Home". Sixtyfive TaskStates were defined for the "buying bread" $\mathrm{ADL}$, including those required to complete de ADL, as well as the variations corresponding to therapeutic interventions, i.e. feedbacks, alternative interactions (e.g.: the patient could pick up keys and wallet in different order), or thera-

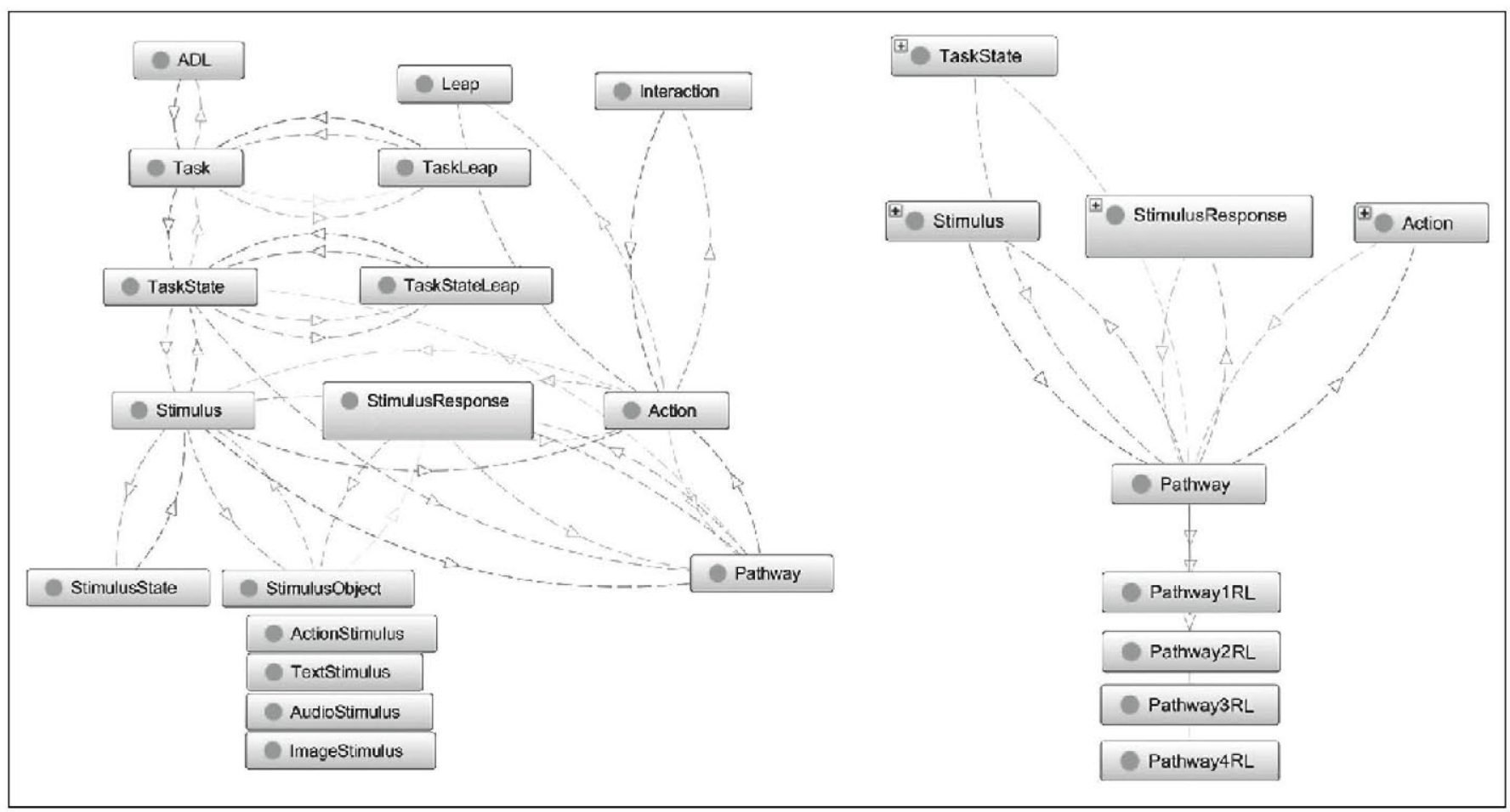

Figure 2 Ontology class diagram: (a) structural level; (b) Pathways-based conceptualization level 


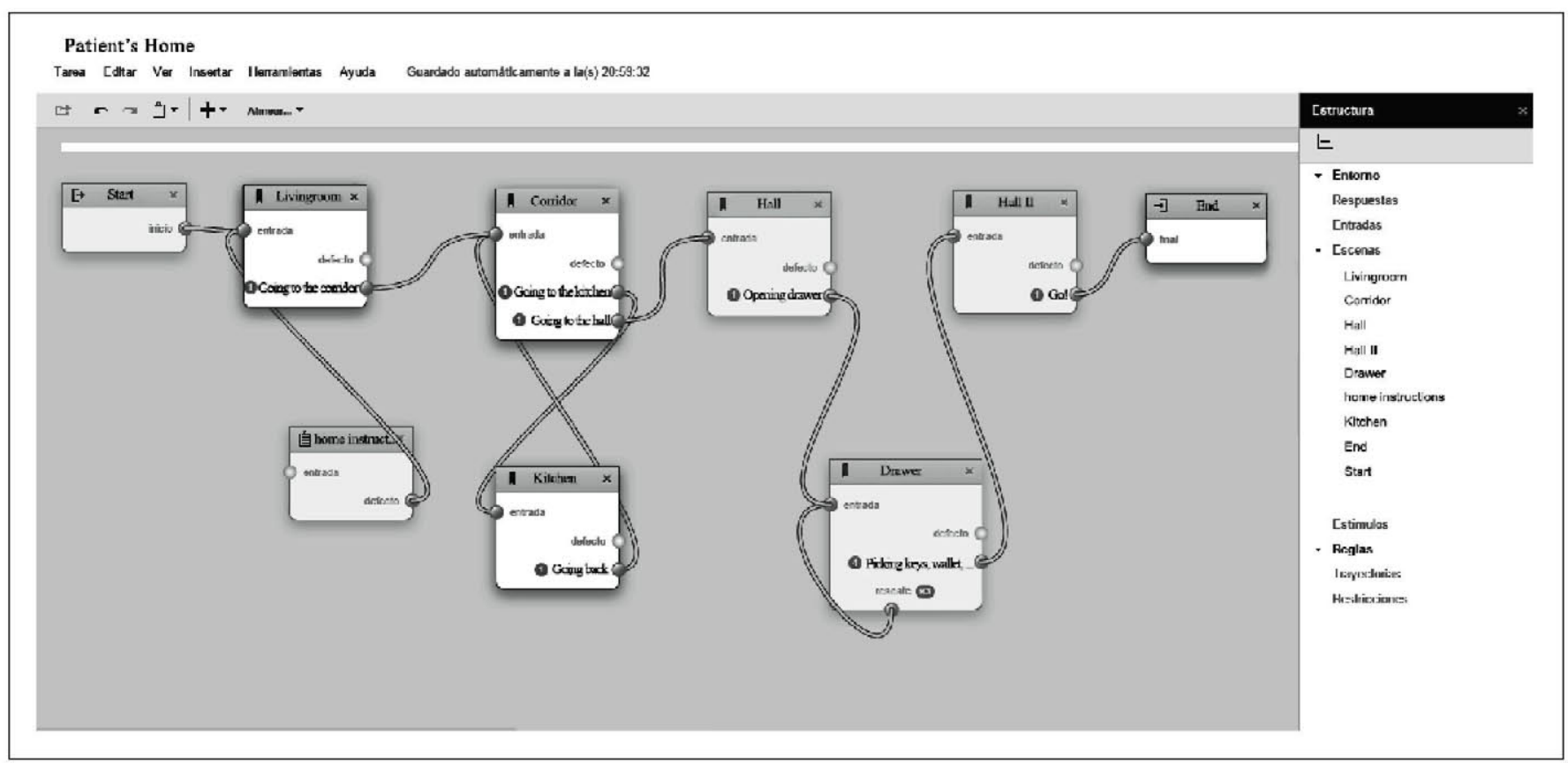

Figure 3 Graphical tool which implements the proposed methodology

peutic supports. Figure 4 shows an example of these therapeutic supports, which aim to help the patient and lead him/her to the right track.

TaskState description involves the definition of every Stimulus which will be shown to the patient, such as visual Stimuli (e.g.: items composing the scenes), instruction Stimuli (e.g.: text showed text or played audios), etc. More than 100 active Stimuli were identified, e.g.: Stimulus "keys" can be picked up using the mouse; two virtual buttons are shown to allow answering a question, etc. Finally, every single interaction has associated a Response Stimuli, in order to specify the shown feedback depending on patient's response, and the transition to the next TaskState (progressing in the ADL or going to a more assisted TaskState). All these elements which define the ADL can be represented by the ontology proposed in the previous section. Table 1 shows an example of each of the ADL's element corresponding to the structural level of the ontology.

According to each Tasks' goals, every single interaction was distinguished between "admissible" and "non-admissible" from a behavioural perspective. Therefore, every defined Pathway could be considered to be represented as belonging to the second Representation Level. As an example of the third Representation Level, some Pathways were designed to cover the same goal but providing a different therapeutic approach. For example, in the Task 'Street' for training of memory function, instructions are given as first TaskState's Stimuli; if attention function is being worked, instructions are given step by step within every TaskState; and for executive functions, instead of text or audio instruction a map is shown.

\section{Discussion}

The present research proposes a methodology to shape ecological cognitive training based on ADLs. The procedure formalizes ADL design based on therapeutic interventions. This enables neuropsychologists to identify the active ingredients in each ADL's stage in order to hypothesise about the patient's behaviour. This approach makes the $\mathrm{ADL}$ into a testing ground, where the neuropsychologist can detect any abnormal behaviour, design intervention strategies and define alternatives in the absence of the expected result. Finally, every single patient's interaction allow clinical experts to define a variety of interventions based on therapeutic hypotheses which will be assessed with every patient's performance, and validated every time the patient successfully complete the ADL.

The methodology is supported by an ADL model whose hierarchical structure allows defining an $\mathrm{ADL}$ splitting it in as many simpler tasks as are required. The methodology allows not only new ADL creation, but also the modification of already existing ADLs, providing neuropsychologists the opportunity to adapt each intervention strategy to the patient's cognitive profile. The final result is an ADL formal description that is independent of the technology that will be used to develop it.

A formal description of an ADL strengthens the integration of the therapies based on $\mathrm{ADL}$ reproduced by interactive virtual environments into routine clinical practice. The proposed methodology aims to bring the activity's structure closer to the clinical professionals' cognitive scheme. Thus, it intends to make these new treatments easier to understand and use, as well as boost their ecological validity and promote their clinical acceptance.

The use of ontologies in ADL-based cognitive rehabilitation encourages research of therapeutic intervention strat- 


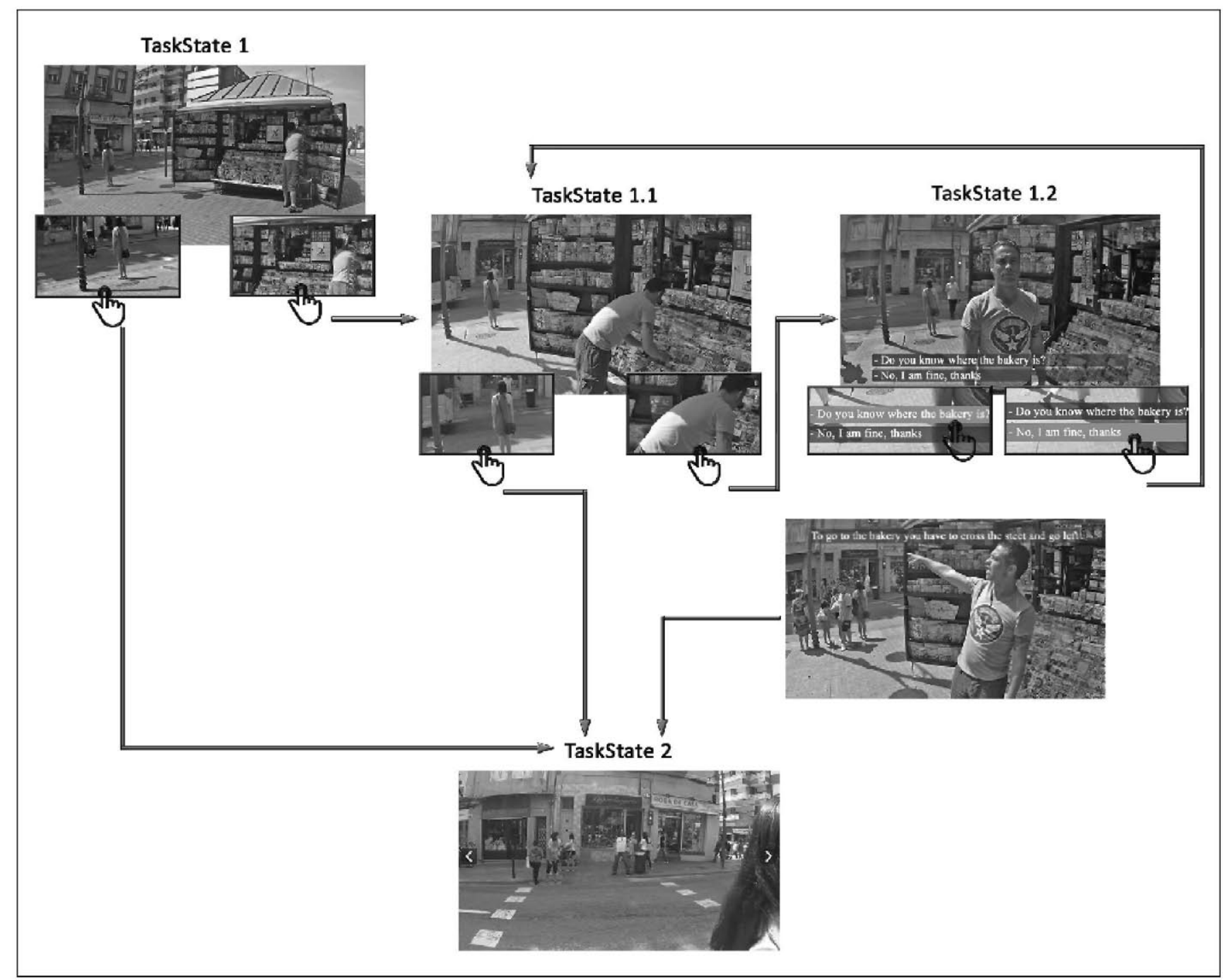

Figure 4 There are different Pathways to achieve TaskState 2 from TaskState 1 depending on the interactions performed by the patients. Whereas the correct interaction will lead the patient immediately to the next TaskState, any other interaction will drive him/her to intermediate TaskStates which contain new stimuli to guide him/her to the correct answer. Interactions in the figure are highlighted by the hand icon.

egies, dissemination of new therapies, and comparison of different alternatives. For that reason, a preliminary approach of the ontology for ADL-based therapeutic interventions has been built to represent our methodology as part of this research work. Further development of the ontology will need to be undertaken in order to integrate it in the scientific community; i.e. mapping with SNOMED CT terminology, connecting with Rehabilitation Treatment Taxonomy [41] or Traumatic Brain Injury Ontology [42].

Technologies such as virtual/augmented reality or interactive video offer many possibilities for the neuropsychologists to set up rehabilitation therapies; however, as the degrees of freedom increase so does the complexity and effort required for designing therapeutic intervention strategies. The proposed methodology may provide clinical professionals access to new complex technologies. In this way, non-technical experts can take the advantages of these technologies applying them to obtain standardized, dynamic and intuitive designs of controlled experiences exploiting the intrinsic property of human cognitive processing to perceive symbolic information as real life experiences.

The ADL "buying bread" was used to assess the feasibility of the proposed meth- odology in the definition of therapeutic intervention procedures. The developed environment reproduces a set of daily situations a person would experience when he/ she is going to buy bread from his/her home. The scenes and the actions mimic those that must be performed in real life, and thus are easily identifiable as familiar situations. However, the user's experience is different than being in a real-life simulator because he/she cannot freely interact with the virtual environment, and when available, interaction is limited by the input devices provided by the technology (in this case, mouse and keyboard). However, the number of defined interactions do not limit 
Table 1

ADL classes according to structural level of the ontology

\begin{tabular}{|c|c|c|c|}
\hline \multicolumn{2}{|l|}{ CLASS } & \multirow[t]{2}{*}{ DESCRIPTION } & \multirow[t]{2}{*}{ EXAMPLE } \\
\hline & SUBCLASS & & \\
\hline \multicolumn{2}{|l|}{$A D L$} & Activity of Daily Living & Buying Bread \\
\hline \multicolumn{2}{|l|}{ Task } & Subactivities of the ADL & Home \\
\hline \multicolumn{2}{|l|}{ TaskState } & $\begin{array}{l}\text { Task's parts which required a patient's in- } \\
\text { teraction }\end{array}$ & Leave Sitting-room \\
\hline \multicolumn{2}{|l|}{ Stimulus } & Minimum unit of information & Hall Door \\
\hline \multicolumn{2}{|l|}{ StimulusState } & State which the stimulus is shown in & Open \\
\hline \multicolumn{2}{|l|}{ StimulusResponse } & $\begin{array}{l}\text { Feedback which patient receives due to } \\
\text { he/she interaction }\end{array}$ & Encourage-Feedback 1 \\
\hline \multirow[t]{4}{*}{ StimulusObject } & ActionStimulus & \multirow[t]{4}{*}{ Kind of element } & VideoClip: leaving the room \\
\hline & ImageStimulus & & Brown Door \\
\hline & TextStimulus & & $\begin{array}{l}\text { Instruction: "Click on the door to leave } \\
\text { the room" }\end{array}$ \\
\hline & AudioStimulus & & Audio Instruction \\
\hline \multicolumn{2}{|l|}{ Action } & $\begin{array}{l}\text { Virtually, what patient is able to do into } \\
\text { the activity }\end{array}$ & Go Through \\
\hline \multicolumn{2}{|l|}{ Interaction } & $\begin{array}{l}\text { Means patients has to interact with the } \\
\text { activity }\end{array}$ & Mouse Click on the Stimulus \\
\hline \multirow[t]{2}{*}{ Leap } & TaskLeap & $\begin{array}{l}\text { Events involves changes in activity flow } \\
\text { Leaps between Tasks }\end{array}$ & $\begin{array}{l}\text { Event: Leap from Home Task to Street } \\
\text { Task }\end{array}$ \\
\hline & TakStateLeap & $\begin{array}{l}\text { Events involves changes in activity flow } \\
\text { Leaps between TaskStates }\end{array}$ & $\begin{array}{l}\text { Event: Leap from leaving sitting-room } \\
\text { TaskState to Hall TaskState }\end{array}$ \\
\hline
\end{tabular}

the ability of the patient to mimic the information with real life situations. The challenge is not to simulate a real environment, because this is part of the mentioned intrinsic processing abilities of the patient, but to perform therapeutic interventions in the patient's ADLs. Keeping the control of what is happening in the activity at every single moment is the essential point to implement a therapeutic intervention strategy. With this purpose, the neuropsychologist defines the interactions that the patient will be allowed to perform, and the stimuli which will encourage him/her. In this way, the cognitive effort required to the patient is adjusted according to his/her cognitive skills.

Converting an unspecific representation of an ADL into a therapeutic intervention will result in an exponential increase of the design and management process difficulty. Even the most simple daily life situation is comprised of great number of stimuli and all possible interactions which would be critical for the final outcome. Thus, following the premise of having under control what is happening in the activity, the neuropsychologist must find a compromise between the complexity of the ADL's definition and the degree of realism experienced by the patient, depending on what is more convenient to the patient's treatment. Moreover, given its potential complexity, it is our belief that non-assisted definition of ADLs is unfeasible, which highlights the importance of the supportive software tool. In this respect, new developments are being carried out to improve the usability and functionality of the tool, in order to provide a higher level of support to the neuropsychologist with the most difficult designs.

The case study "buying bread" ADL proves the potential of the proposed methodology to design therapeutic intervention strategies. However, more experiments must be done in order to present more evidence about the values, advantages and constraints of the methodology applicability. Designing new ADL experiments could show if the proposed framework is useful in assessing the effectiveness of the therapies and is also able to help improve them.

An empirical study is being designed with the aim of assessing subjects' behaviour according to the therapeutics hypothesis used to define the ADL "buying bread". The registered information during the performance of the ADL will be combined with their visual attention information, monitored by an eye-tracking system. The empirical study will be carried out in the Institut Guttmann Hospital.

\section{Conclusions}

This research work proposes a new methodology for shaping ecological cognitive BI rehabilitation. There are currently different approaches to the use of therapeutic virtual environments to reproduce ADLs, but nowadays they are not integrated into routine clinical practice. The proposed methodology provides neuropsychologists with an instrument to design ADL-based therapeutic intervention strategies, attending to 
fill the gap between clinical knowledge and the ADL well-definition.

An ADL Intervention Model was developed to support the methodology. The ADL "buying bread" was defined according to the model's guidelines and developed using interactive video technology. It is currently being used to assess the feasibility of the proposed methodology in the definition of therapeutic intervention procedures. With that purpose, an empirical study is being designed and it will be carry out in the Institut Guttmann Hospital.

\section{Acknowledgments}

The authors thank Ignacio Oropesa from the Biomedical Engineering and Telemedicine Centre for his collaboration with this research work. We also thank Samuel González from Lavinia Interactiva for his collaboration especially with the development of the graphical tool. This research has been partially founded by the Spanish Ministry of Economy and Finance (project TIN2012-38450, COGNITIO).

\section{References}

1. Elbaum J. Acquired Brain Injury and the Family. Acquired Brain Injury: Springer; 2007. pp 275-285.

2. Barbara Woodward Lips Patient Education Center. Understanding Brain Injury. A Guide for the Family. 2008.

3. Karol RL. Neuropsychosocial intervention: The practical treatment of severe behavioral dyscontrol after acquired brain injury. CRC press; 2003.

4. Marcotte TD, Cobb Scott J, Kamat R, Heaton RK. Neuropsychology and the Prediction of Everyday Functioning. In: Marcotte TD, Grant I, editors. Neuropsychology of Everyday Functioning. The Guilford Press; 2010. pp 5-38.

5. Kaplan RM, Mausbach BT, Marcotte TD, Patterson TL. The Impact of Cognitive Impairments on Healh-Related Quality of Life. In: Marcotte TD, Grant I, editors. Neuropsychology of Everyday Functioning. The Guilford Press; 2010. pp 225-247.

6. Wilson BA. Cognitive Rehabilitation: How it is and how it might be. J Int Neuropsychol Soc 1997; 3 (5): 487- 496

7. Lövdén M, Bäckman L, Lindenberger U, Schaefer S, Schmiedek F. A theoretical framework for the study of adult cognitive plasticity. Psychol Bull 2010; $136(4): 659$.

8. Pascual-Leone A, Amedi A, Fregni F, Merabet L. The plastic human brain cortex. Annu Rev Neurosci 2005 ; 28 : $377-401$.

9. Johansson BB. Current trends in stroke rehabilitation. A review with focus on brain plasticity. Acta Neurol Scand 2011; 123 (3): 147-159.
10. Kleim JA, Jones TA. Principles of experience-dependent neural plasticity: implications for rehabilitation after brain damage. Journal of Speech, Language, and Hearing Research 2008; 51 (1): S225-S239.

11. Cicerone KD, Langenbahn DM, Braden C, Malec JF, Kalmar K, Fraas M, et al. Evidence-Based Cognitive Rehabilitation: Updated Review of the Literature from 2003 through 2008. Arch Phys Med Rehabil 2011; 92 (4): 519-530.

12. Rizzo AA, Schultheis M, Kerns KA, Mateer C. Analysis of assets for virtual reality applications in neuropsychology. Neuropsychological Rehabilitation 2004; 14 (1-2): 207-239.

13. Green CS, Bavelier D. Exercising Your Brain: A Review of Human Brain Plasticity and TrainingInduced Learning. Psychol Aging 2008; 23 (4): 692-701.

14. Wilson BA. Neuropsychological rehabilitation: State of the science. S Afr J Psychol 2013; 43 (3): 267-277.

15. Wilson BA, Herbert CM, Shiel A. Behavioural approaches in neuropsychological rehabilitation: Optimising rehabilitation procedures. Psychology Press; 2003.

16. Solana Sanchez J, Caceres C, García-Molina A, Opisso E, Roig T, Tormos J, et al. Improving brain injury cognitive rehabilitation by personalized tele-rehabilitation services: Guttmann Neuro Personal Trainer system. 2014.

17. Marcano-Cedeño A, Chausa P, García A, Cáceres C, Tormos JM, Gómez EJ. Artificial metaplasticity prediction model for cognitive rehabilitation outcome in acquired brain injury patients. Artif Intell Med 2013; 58 (2): 91-99.

18. Marcano-Cedeño A, Chausa P, García A, Cáceres C, Tormos JM, Gómez EJ. Data mining applied to the cognitive rehabilitation of patients with acquired brain injury. Expert Syst Appl 2013; 40 (4): $1054-1060$.

19. Aghajan Z M, Acharya L, Moore J J, Cushman J D, Vuong C, Mehta M R. Impaired spatial selectivity and intact phase precession in two-dimensional virtual reality. Nature Neuroscience 2015; 18: 121-128.

20. Katz N, Ring H, Naveh Y, Kizony R, Feintuch U, Weiss PL. Interactive virtual environment training for safe street crossing of right hemisphere stroke patients with Unilateral Spatial Neglect. Disabil Rehabil 2005; 27 (20): 1235-1243.

21. Edmans J, Gladman J, Walker M, Sunderland A, Porter A, Fraser DS. Mixed reality environments in stroke rehabilitation: Development as rehabilitation tools. International Journal on Disability and Human Development 2004; 6 (1): $39-45$.

22. Sorita E, N'Kaoua B, Larrue F, Criquillon J, Simion A, Sauzéon $H$, et al. Do patients with traumatic brain injury learn a route in the same way in real and virtual environments? Disabil Rehabil 2013 35 (16): 1371-1379.

23. Rizzo AA, Bowerly T, Buckwalter JG, Klimchuk D, Mitura R, Parsons TD. A virtual reality scenario for all seasons: The virtual classroom. Cns Spectrums 2006; 11 (1): 35 - 44 .

24. Renison B, Ponsford J, Testa R, Richardson B, Brownfield $\mathrm{K}$. The Ecological and Construct Validity of a Newly Developed Measure of Executive Function: The Virtual Library Task. J Int Neuropsychol Soc 2012; 18 (3): 440 - 450.
25. Tarnanas I, Tsolakis A, Tsolaki M. Assessing Virtual Reality Environments as Cognitive Stimulation Method for Patients with MCI. Technologies of Inclusive Well-Being. Springer; 2014. pp 39-74.

26. Fidopiastis CM, Stapleton CB, Whiteside JD, Hughes CE, Fiore SM, Martin GA, et al. Human experience modeler: Context-driven cognitive retraining to facilitate transfer of learning. CyberPsychology \& Behavior 2006; 9 (2): 183 - 187.

27. Klinger E, Cao X, Douguet A, Fuchs P. Designing an Ecological and Adaptable Virtual Task in the Context of Executive Functions. Cyberpsychology \& Behavior 2009; 12 (5): 626-627.

28. Gamito P, Oliveira J, Caires C, Morais D, Brito R, Lopes P, et al. Virtual Kitchen Test Assessing Frontal Lobe Functions in Patients with Alcohol Dependence Syndrome. Methods Inf Med 2015; 54 (2): $122-126$

29. da Costa R, de Carvalho L, de Aragon D. Virtual city for cognitive rehabilitation. Proceedings of the 3rd International Conference on Disability Virtual Reality and Associated Technologies. Alghero, Sardinia; 2000

30. Dores A, Miranda M, Carvalho I, Mendes L, Barbosa F, Coelho A, de Sousa L, Caldas A. Virtual City: Neurocognitive rehabilitation of Acquired Brain Injury. Information Systems and Technologies (CISTI), 2012 7th Iberian Conference on: IEEE; 2012

31. Klinger E, Kadri A, Sorita E, Le Guiet J, Coignard P, Fuchs P, et al. AGATHE: A tool for personalized rehabilitation of cognitive functions based on simulated activities of daily living. IRBM 2013; 34 (2): 113-118.

32. Grewe P, Kohsik A, Flentge D, Dyck E, Botsch M, Winter Y, et al. Learning real-life cognitive abilities in a novel 360 degrees-virtual reality supermarket: a neuropsychological study of healthy participants and patients with epilepsy. J NeuroEng Rehabil 2013; 10: 42 .

33. Lee JH, Ku J, Cho W, Hahn WY, Kim IY, Lee S, et al. A virtual reality system for the assessment and rehabilitation of the activities of daily living. CyberPsychology \& Behavior 2003; 6 (4): 383-388.

34. Werner P, Rabinowitz S, Klinger E, Korczyn AD, Josman N. Use of the virtual action planning supermarket for the diagnosis of mild cognitive impairment: a preliminary study. Dement Geriatr Cogn Disord 2009; 27 (4): 301-309.

35. Rand D, Weiss PL(, Katz N. Training Multitasking in a Virtual Supermarket: A Novel Intervention After Stroke. Am J Occup Ther 2009; 63 (5): 535-542.

36. Pietrzak E, Pullman S, McGuire A. Using Virtual Reality and Videogames for Traumatic Brain Injury Rehabilitation: A Structured Literature Review. GAMES FOR HEALTH: Research, Development, and Clinical Applications 2014; 3 (4): 202-214.

37. Riva G, Gaggioli A, Grassi A, Raspelli S, Cipresso P, Pallavicini F, et al. NeuroVR 2 - a free virtual reality platform for the assessment and treatment in behavioral health care. Stud Health Technol Inform 2011; 163: 493-495.

38. Lange B, Koenig S, Chang C, McConnell E, Suma E, Bolas M, et al. Designing informed game-based rehabilitation tasks leveraging advances in virtual reality. Disabil Rehabil 2012; 34 (22): 1863 -1870. 
39. Whyte J, Hart T. It's more than a black box; It's a Russian doll - Defining rehabilitation treatments. Am J Phys Med Rehabil 2003; 82 (8): 639- 652 .

40. Whyte J, Dijkers MP, Hart T, Zanca JM, Packel A, Ferraro M, et al. Development of a theory-driven rehabilitation treatment taxonomy: conceptual issues. Arch Phys Med Rehabil 2014; 95 (1): S24-S32. e2.

41. Dijkers MP, Hart T, Tsaousides T, Whyte J, Zanca JM. Treatment Taxonomy for Rehabilitation: Past, Present, and Prospects. Arch Phys Med Rehabil 2014; 95 (1): S6-S16.

42. Grove MJ. Development of an Ontology for Rehabilitation: Traumatic Brain Injury. University of Minnesota; 2013

43. Giustini A, Varela E, Franceschini M, Votava J, Zampolini M, Berteanu M, et al. UEMS--Position
Paper. New technologies designed to improve functioning: the role of the physical and rehabilitation medicine physician. European journal of physical and rehabilitation medicine 2014; 50 (5) $579-583$.

44. Baum CM, Katz N. Occupational Therapy Approach to Assessing the Relationship between Cognition and Function. In: Marcotte TD, Grant I editors. Neuropsychology of Everyday Functioning. The Guilford Press; 2010. pp 62-90.

45. Stanford University. Protégé. 2014

46. W3C OWL Working Group. OWL 2 Web Ontology Language Document Overview (Second Edition). 2012; Available at: http://www.w3.org/TR/ owl2-overview/. Accessed September, 2014.

47. McGuinness D, Van Harmelen F. OWL Web Ontology Language Overview. 2004; Available at: http://www.w3.org/standards/techs/owl\#w3c_all. Accessed September, 2014.

48. Martínez-Moreno JM, Sánchez-González P, García A, González S, Cáceres C, Sánchez-Carrión R, Roig T, Tormos JM, Gómez EJ. A Graphical Tool for Designing Interactive Video Cognitive Rehabilitation Therapies. XIII Mediterranean Conference on Medical and Biological Engineering and Computing 2013. Springer; 2014.

49. Martinez-Moreno JM, Solana J, Sanchez R, Gonzalez S, Sanchez-Gonzalez P, Gomez C, et al. Cognitive Neurorehabilitation based on Interactive Video Technology. Informatics, Management and Technology in Healthcare 2013; 190: 27-29.

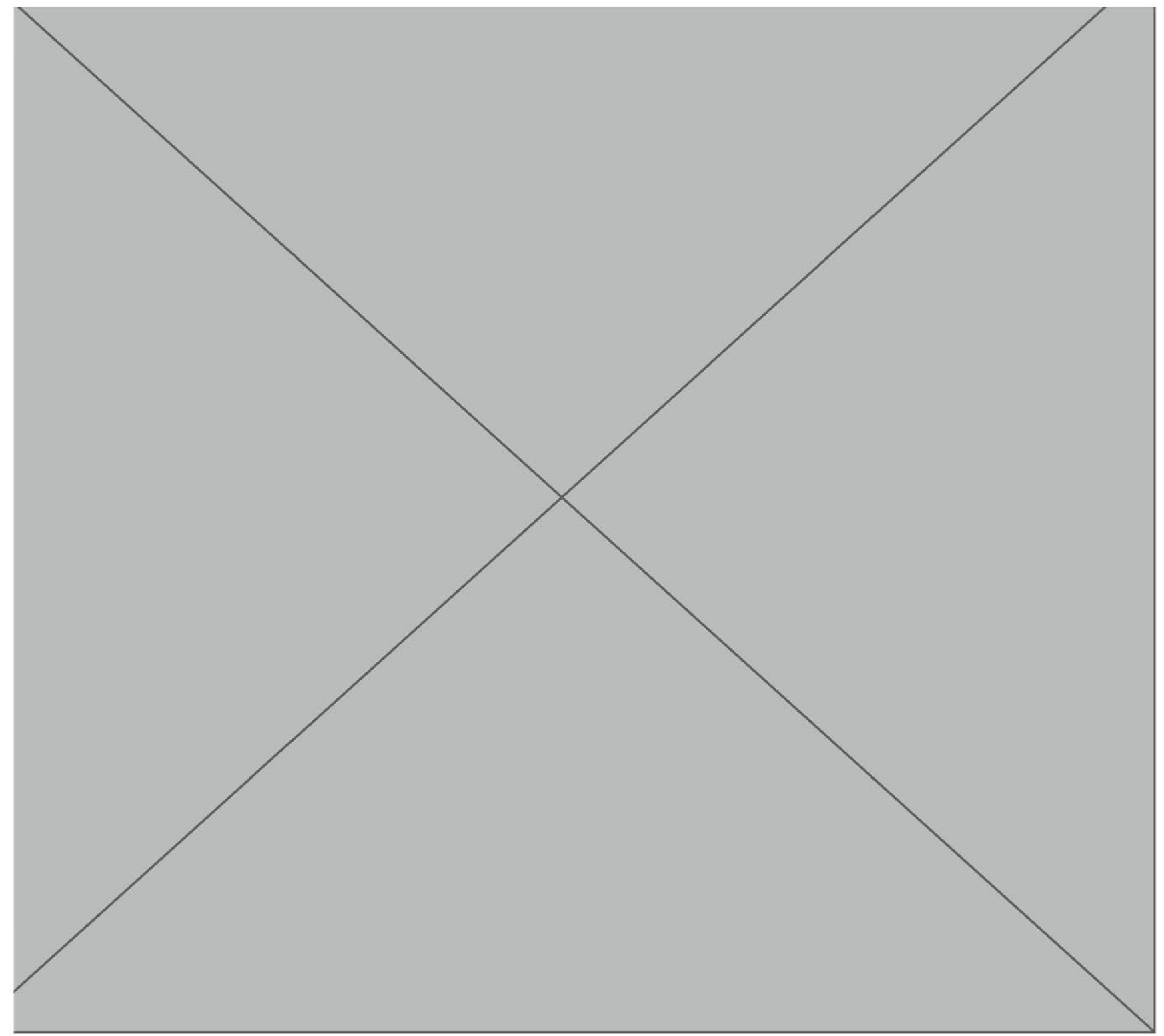

